\title{
Blue Laser Imaging with a Small-Caliber Endoscope Facilitates Detection of Early Gastric Cancer
}

\author{
Haruo Takahashi', Yoshimasa Miura ${ }^{1}$, Hiroyuki Osawa ${ }^{1}$, Takahito Takezawa ${ }^{1}$, Yuji Ino ${ }^{1}$, Masahiro 0 kada $^{1}$, Alan Kawarai Lefor ${ }^{2}$ and \\ Hironori Yamamoto ${ }^{1}$ \\ ${ }^{1}$ Division of Gastroenterology, Department of Medicine, ${ }^{2}$ Department of Surgery, Jichi Medical University, Shimotsuke, Tochigi, Japan
}

Conventional endoscopy often misses early gastric cancers with minimal red discoloration because they cannot be distinguished from inflamed mucosa. We treated a patient with a small early gastric cancer that was difficult to diagnose using conventional endoscopy. Conventional endoscopy using a small-caliber endoscope showed only subtle red discoloration of the gastric mucosa. However, blue laser imaging showed a clearly discolored area measuring $10 \mathrm{~mm}$ in diameter around the red lesion, which was distinct from the surrounding inflamed mucosa. Irregular vessels on the tumor surface (suspicious for early gastric cancer) were observed even with small-caliber endoscopy. Biopsy revealed a well-moderately differentiated tubular adenocarcinoma, and endoscopic submucosal dissection was performed. Histopathological examination of the specimen confirmed well-moderately differentiated adenocarcinoma localized to the mucosa with slight depression compared to the surrounding mucosa, consistent with the endoscopic findings. This small early gastric cancer became clearly visible with blue laser imaging using small-caliber endoscopy. Clin Endosc 2019;52:273-277

Key Words: Early gastric cancer; Blue laser imaging; Small-caliber endoscopy; Endoscopic submucosal dissection

\section{INTRODUCTION}

Early detection of gastric cancer is difficult even among well-trained endoscopists, leading to frequently missed lesions. ${ }^{1}$ Although the accurate diagnosis and growth characteristics of gastrointestinal lesions are well described because of the development of magnifying and image-enhanced endoscopy, the usefulness of early detection through screening remains unclear. Recently, a new laser endoscopic system has been developed, which includes linked color imaging (LCI) and blue laser imaging (BLI), which are receiving a great deal of attention for the early detection of gastrointestinal

Received: June 9, 2018 Revised: July 3, 2018

Accepted: July 4, 2018

Correspondence: Hiroyuki Osawa

Division of Gastroenterology, Department of Medicine, Jichi Medical University, 3311-1 Yakushiji, Shimotsuke, Tochigi 329-0498, Japan

Tel: +81-285-58-7539, Fax: +81-285-44-0047, E-mail: osawa@jichi.ac.jp

ORCID: https://orcid.org/0000-0002-1479-0475

cc This is an Open Access article distributed under the terms of the Creative Commons Attribution Non-Commercial License (http://creativecommons.org/ licenses/by-nc/3.0) which permits unrestricted non-commercial use, distribution, and reproduction in any medium, provided the original work is properly cited. cancers. ${ }^{2,3}$ LCI provides color information regarding the red, green and blue components and provides an expanded color range, which causes red and white colors to become redder and whiter, respectively. ${ }^{2,5,5} \mathrm{LCI}$ facilitates easier recognition of even small differences in mucosal color, although the mucosal colors obtained by LCI are similar to those obtained using white light imaging. BLI provides color information regarding the green and blue components and provides high color contrast between malignant lesions and the surrounding mucosa, in near and close-up views. ${ }^{6,7}$

Endoscopists may occasionally face difficulties with identifying gastric lesions, particularly those located on the posterior gastric wall and/or near the gastric angle. The degree of difficulty varies depending upon the various kinds of endoscopes used. A few gastric cancers are difficult to identify in tangential views using a normal-caliber endoscope under direct vision, resulting in missed lesions. A small-caliber endoscope has a small radius at the tip of the endoscope, which allows observation of upper gastrointestinal lesions in a frontal view. ${ }^{8}$ Small-caliber endoscopy has been reported to provide poor quality endoscopic images compared with normal caliber 
endoscopy. ${ }^{9-11}$ However, recent progress with the development of improved technology can deliver high-resolution images. LCI and BLI with a small-caliber endoscope (EG-L580NW; Fujifilm, Tokyo, Japan) using a laser endoscopic system have been available since 2014. However, whether LCI and BLI using a small-caliber endoscope can yield findings similar to normal-caliber endoscopy for gastrointestinal lesions remains undetermined, although it is commonly used in Japan to screen for upper gastrointestinal lesions.

We report a patient with an early gastric cancer of the posterior wall near the gastric angle, which was difficult to diagnose using only white light imaging, as well as a normal-caliber endoscope at the time of screening. BLI with a small-caliber endoscope was used in this patient.

\section{CASE REPORT}

A 79-year-old man underwent esophagogastroduodenoscopy with a small-caliber endoscope (EG-L580NW; Fujifilm) using a laser endoscopic system as part of a medical examination at a Neurology clinic. He complained of slight epigastric bloating. He reported a history of hypertension and was prescribed an antihypertensive drug. He reported no other symptoms at the time of the esophagogastroduodenoscopy. Laboratory tests showed anti-Helicobacter pylori antibody 6.4 IU (positive) and a positive stool antigen test for H. pylori. Other blood and chemistry tests showed normal results. Abdominal ultrasonography and a computed tomography scan showed no abnormal findings. White light imaging with a small-caliber endoscope showed a red area measuring $3 \mathrm{~mm}$ in diameter on the posterior gastric wall near the gastric angle (Fig. 1A), which was not suspicious for gastric cancer based on its appearance. LCI enhanced the red lesion and the surrounding red area (Fig. 1B). BLI revealed a discolored lesion measuring $10 \mathrm{~mm}$ around a central red area with an apparent demarcation between the cancer and the surrounding mucosa with high color contrast (Fig. 1C, D). Several irregular vessels were observed on the discolored lesion even with the use of small-caliber endoscopy, suggesting an early gastric cancer. The biopsy showed well-moderately differentiated tubular adenocarcinoma. The patient was referred to Jichi Medical University Hospital for further evaluation and treatment.

Laser endoscopy was performed using a transoral endoscope
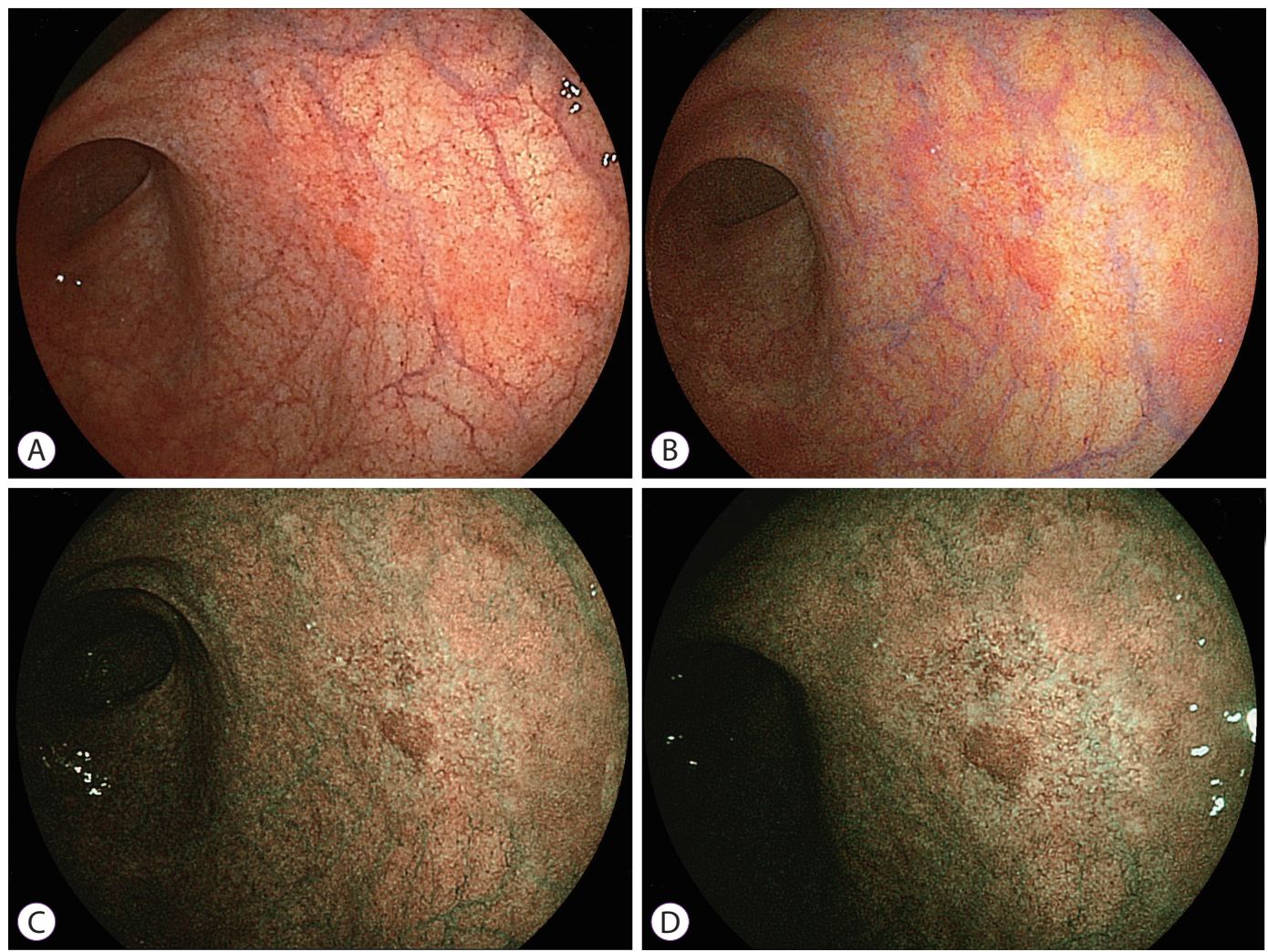

Fig. 1. (A) White light imaging with a small-caliber endoscope shows a small red area measuring $3 \mathrm{~mm}$ in diameter on the posterior wall near the gastric angle, which is not suspicious for gastric cancer. (B) Linked color imaging enhances the red lesion and the surrounding red portion. (C) Bright blue laser imaging reveals a discolored lesion measuring $10 \mathrm{~mm}$ around a central red area. (D) Blue laser imaging produces a high color contrast between the malignant lesion and the surrounding mucosa. Several irregular vessels are seen in the discolored lesion even with small-caliber endoscopy, suggesting early gastric cancer. 
(EG-L590ZW; Fujifilm); however, the lesion could not be clearly visualized without magnification because of tangential views obtained (Fig. 2A, B). Magnified BLI imaging showed an apparent demarcation between the slightly depressed cancer and the surrounding mucosa, as well as irregular microvessels and microstructure distinct from the surrounding mucosa (Fig. 2C, D). Endoscopic ultrasound revealed cancer limited to the mucosa. Two months later, marks were placed $5 \mathrm{~mm}$ outside the demarcation line, and endoscopic submucosal dissection was performed (Fig. 3A). The resected specimen showed a slightly depressed early gastric cancer with well-moderately differentiated tubular adenocarcinoma limited to the mucosa (Fig. 3B, C). Additionally, microvessels were identified in the intervening part of the superficial layer of the mucosa (Fig. 3D), supporting the identification of vessels with BLI using small-caliber endoscopy.

\section{DISCUSSION}

BLI performed with a small-caliber endoscope allows detection of features of early gastric cancer that are difficult to detect using white light imaging. Although white light imaging showed only a small red area, BLI showed a larger discolored lesion around it with irregular vessels suspicious for early gastric cancer. Additionally, this endoscope allowed us to observe the lesion under direct vision despite its location on the posterior wall near the gastric angle. Small-caliber endoscopy using BLI provided accurate and clear images, even during routine health examination.

It is difficult for many endoscopists to recognize the subtle color changes in the gastric mucosa as a definitive cancer. ${ }^{12}$ Most gastric cancers are surrounded by chronically inflamed mucosa with atrophic changes and intestinal metaplasia. ${ }^{13-15}$ These surrounding features make it difficult to identify the demarcation between a malignant lesion and the surrounding mucosa. Recently, laser endoscopy has been developed and is reported to be useful for the diagnosis of a few gastrointestinal lesions, ${ }^{3,16-20}$ although to date, no studies have reported using small-caliber laser endoscopy.

In this patient, BLI allowed detection of an early gastric cancer with a clearly apparent demarcation, which could not be observed using white light imaging. Many endoscopists in Japan use a small-caliber endoscope to screen patients for
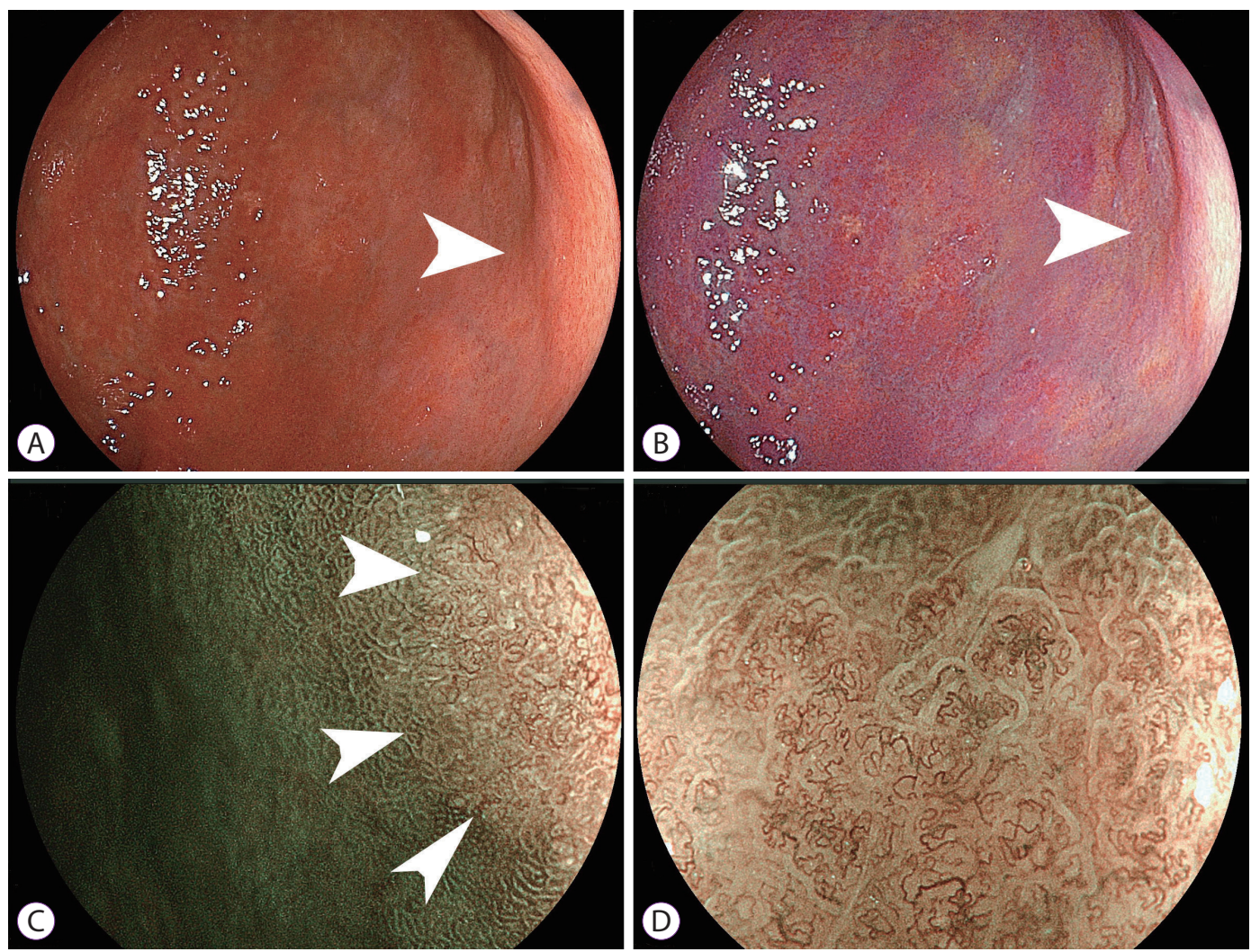

Fig. 2. Images obtained with a normal-caliber endoscope: (A) white light imaging and (B) linked color imaging cannot clearly reveal the site of the early gastric cancer (white arrows) because of the tangential view. (C) Blue laser imaging with middle magnification shows a brown malignant lesion surrounded by green mucosa (white arrows). (D) Blue laser imaging using high magnification shows irregular microvascular and irregular microstructural patterns on the mucosal surface. 

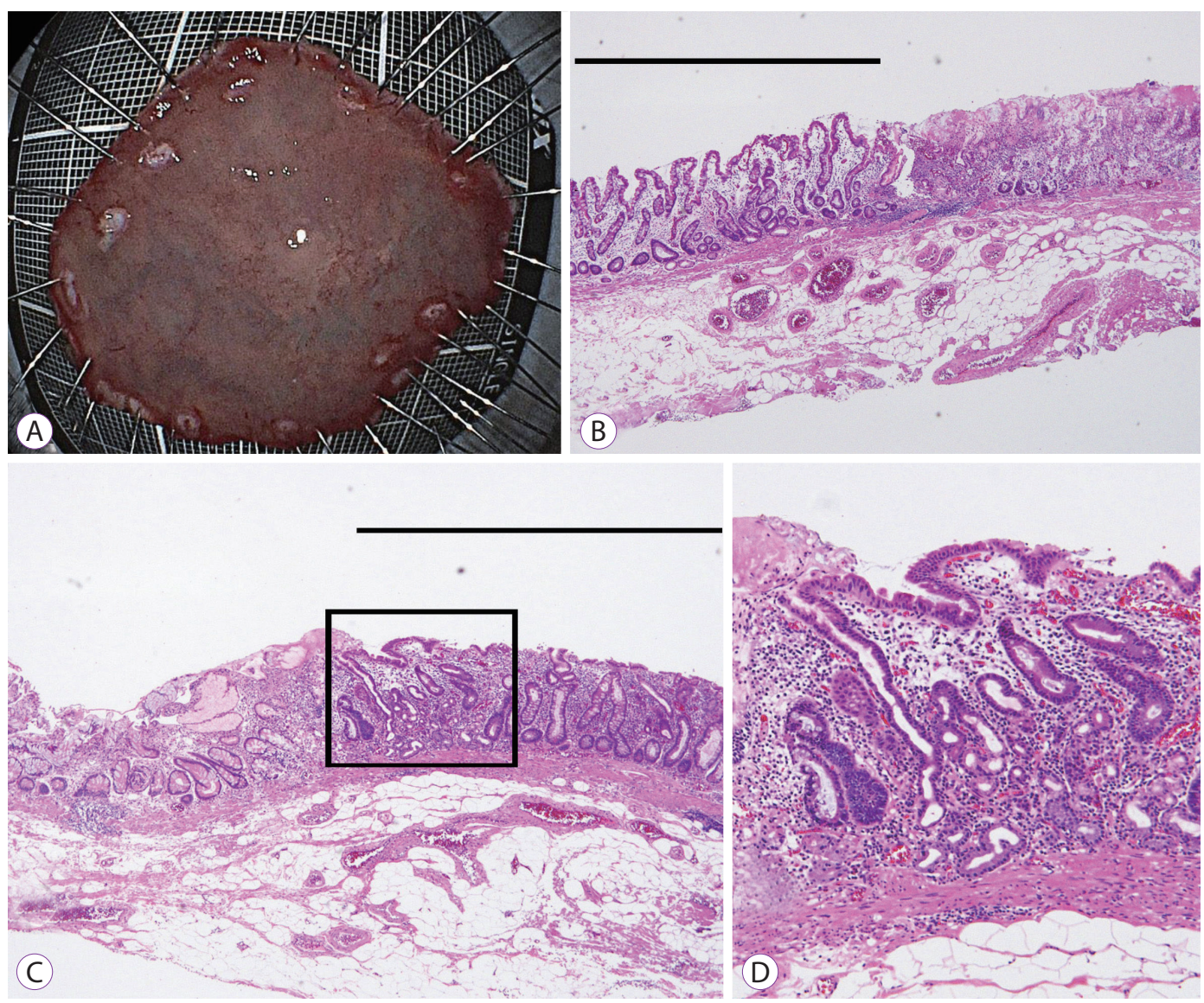

Fig. 3. (A) The specimen resected by endoscopic submucosal dissection can be observed. (B) Histopathological evaluation of the resected specimen shows well-differentiated adenocarcinoma (black line) (hematoxylin \& eosin [H\&E], $\times 40$ ) and (C) moderately-differentiated adenocarcinoma (black line) (H\&E, $\times 40)$. (D) Magnified imaging of the square in $(C)$ shows that microvessels are present in the intervening part of the superficial layer of the mucosa $(H \& E, \times 100)$.

upper gastrointestinal lesions. The endoscope with a diameter measuring $5.9 \mathrm{~mm}$ causes minimal pain and gag reflex during the endoscopic examination. Using the laser endoscopic system, small-caliber endoscopy produces bright and high-resolution white light images that are suitable for screening patients for upper gastrointestinal lesions. However, in this patient, white light imaging did not reveal definitive findings of gastric cancer. Most areas of cancer showed a color similar to the surrounding mucosa using white light imaging, whereas BLI showed a different color. During screening examinations, endoscopists must not depend only on conventional color patterns using white light imaging to avoid missing upper gastrointestinal lesions. Large differences in color between malignant lesions and the surrounding mucosa lead us to evaluate suspicious areas more carefully. Recently, LCI has been used to screen patients for early gastric cancers ${ }^{2,5}$ and showed an enhanced malignant lesion in this case as well. Additionally, the results of this case report suggest that BLI may also be suitable to identify early gastric cancers in a few areas of the stomach such as antrum or near angle where brighter images are not required.

Small-caliber laser endoscopes have a short focal length of 3 $\mathrm{mm}$ and thus allow close-up views. Laser endoscopy produces a combined image created using spectral and white light imaging. BLI has a high emission intensity at $410 \mathrm{~nm}$ (short wavelength) reflecting on the mucosal surface in addition to low intensity white light imaging. These characteristics may be useful to enhance the visibility of details on the tumor surface, even with small-caliber endoscopy. In this patient, using BLI, an irregular vascular pattern was visualized in the malignant portion but not in the surrounding mucosa. The small radius of the endoscope tip enables endoscopists to observe lesions near the gastric angle under direct vision. A normal-caliber endoscope may occasionally not show lesions in this area because of an inappropriate distance between the lesion and the tip of the endoscope. These characteristics of small-caliber endoscopy also help to distinguish malignant from non-malignant lesions.

This report describes the usefulness of LCI and BLI with a small-caliber endoscope to establish the diagnosis of an early 
gastric carcinoma. A large prospective controlled trial is warranted to demonstrate the usefulness of BLI with a small-caliber endoscope for the diagnosis of early gastric cancer. In conclusion, white light imaging using small-caliber endoscopy may not allow the diagnosis of a small red lesion as cancer; however, BLI could show a discolored area around the lesion with irregular vessels distinct from the surrounding non-malignant area, allowing the accurate diagnosis of an early gastric cancer.

\section{Conflicts of Interest}

Hiroyuki Osawa received commercial research funding from Fujifilm Medical Corporation. Hironori Yamamoto has been a consultant with Fujifilm Corporation and has received honoraria, grants, and royalties from the company. The other authors have no financial conflicts of interest.

\section{REFERENCES}

1. Raftopoulos SC, Segarajasingam DS, Burke V, Ee HC, Yusoff IF. A cohort study of missed and new cancers after esophagogastroduodenoscopy. Am J Gastroenterol 2010;105:1292-1297.

2. Fukuda H, Miura Y, Hayashi Y, et al. Linked color imaging technology facilitates early detection of flat gastric cancers. Clin J Gastroenterol 2015;8:385-389.

3. Yoshida N, Yagi N, Inada Y, et al. Ability of a novel blue laser imaging system for the diagnosis of colorectal polyps. Dig Endosc 2014;26:250258.

4. Okada M, Sakamoto H, Takezawa T, et al. Laterally spreading tumor of the rectum delineated with linked color imaging technology. Clin Endosc 2016;49:207-208.

5. Dohi O, Yagi N, Onozawa $Y$, et al. Linked color imaging improves endoscopic diagnosis of active Helicobacter pylori infection. Endosc Int Open 2016;4:E800-E805.

6. Osawa H, Yamamoto H, Miura Y, et al. Blue laser imaging provides excellent endoscopic images of upper gastrointestinal lesions. Video Journal and Encyclopedia of GI Endoscopy 2014;1:607-610.

7. Osawa H, Yamamoto H. Present and future status of flexible spectral imaging color enhancement and blue laser imaging technology. Dig Endosc 2014;26 Suppl 1:105-115.

8. Toma S, Osawa H, Yoshizawa M, et al. Diagnosis of small flat early gastric cancer by flexible spectral imaging color enhancement. Clin J Gastroenterol 2010;3:88-91.

9. Zaman A, Hahn M, Hapke R, Knigge K, Fennerty MB, Katon RM. A randomized trial of peroral versus transnasal unsedated endoscopy using an ultrathin videoendoscope. Gastrointest Endosc 1999;49(3 Pt 1):279-284.

10. Birkner B, Fritz N, Schatke W, Hasford J. A prospective randomized comparison of unsedated ultrathin versus standard esophagogastroduodenoscopy in routine outpatient gastroenterology practice: does it work better through the nose? Endoscopy 2003;35:647-651.

11. Faulx AL, Catanzaro A, Zyzanski S, et al. Patient tolerance and acceptance of unsedated ultrathin esophagoscopy. Gastrointest Endosc 2002;55:620-623

12. Mori M, Adachi Y, Kakeji Y, et al. Superficial flat-type early carcinoma of the stomach. Cancer 1992;69:306-313.

13. Nakamura K, Sugano H, Takagi K. Carcinoma of the stomach in incipient phase: its histogenesis and histological appearances. Gan 1968;59:251-258.

14. Matsukura N, Suzuki K, Kawachi T, et al. Distribution of marker enzymes and mucin in intestinal metaplasia in human stomach and relation to complete and incomplete types of intestinal metaplasia to minute gastric carcinomas. J Natl Cancer Inst 1980;65:231-240.

15. Kohli Y, Pfeiffer CJ, Kutty KP, Barrowman JA, Heughan C, Kepkay DL. Endoscopic diagnosis of intestinal metaplasia in Canada and Japan. J Clin Gastroenterol 1981;3 Suppl 1:29-33.

16. Yoshida N, Hisabe T, Inada Y, et al. The ability of a novel blue laser imaging system for the diagnosis of invasion depth of colorectal neoplasms. J Gastroenterol 2014;49:73-80.

17. Dohi O, Yagi N, Majima A, et al. Diagnostic ability of magnifying endoscopy with blue laser imaging for early gastric cancer: a prospective study. Gastric Cancer 2017;20:297-303.

18. Ikematsu H, Sakamoto T, Togashi K, et al. Detectability of colorectal neoplastic lesions using a novel endoscopic system with blue laser imaging: a multicenter randomized controlled trial. Gastrointest Endosc 2017;86:386-394.

19. Tomie A, Dohi O, Yagi N, et al. Blue laser imaging-bright improves endoscopic recognition of superficial esophageal squamous cell carcinoma. Gastroenterol Res Pract 2016;2016:6140854.

20. Iwashita C, Miura $\mathrm{Y}$, Osawa $\mathrm{H}$, et al. Laser imaging facilitates early detection of synchronous adenocarcinomas in patients with Barrett's esophagus. Clin Endosc 2017;50:81-86. 